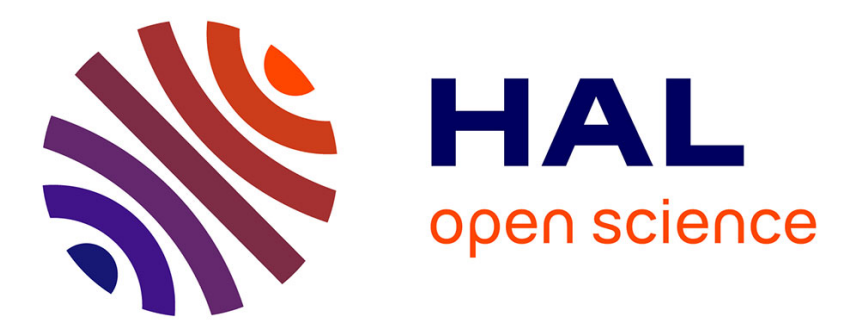

\title{
Chlamydiose et fièvre $\mathrm{Q}$ chez les ongulés de montagne: questions sur le seuil de positivité
}

Emmanuelle Gilot-Fromont, Dominique Gauthier, Philippe Gibert, Jean Hars, Michel Catusse

\section{- To cite this version:}

Emmanuelle Gilot-Fromont, Dominique Gauthier, Philippe Gibert, Jean Hars, Michel Catusse. Chlamydiose et fièvre Q chez les ongulés de montagne: questions sur le seuil de positivité. Médecine et Maladies Infectieuses, 2001, pp.311-312. 10.1016/S0399-077X(01)80080-9 . hal-00427131

\section{HAL Id: hal-00427131 https://hal.science/hal-00427131}

Submitted on 17 May 2021

HAL is a multi-disciplinary open access archive for the deposit and dissemination of scientific research documents, whether they are published or not. The documents may come from teaching and research institutions in France or abroad, or from public or private research centers.
L'archive ouverte pluridisciplinaire HAL, est destinée au dépôt et à la diffusion de documents scientifiques de niveau recherche, publiés ou non, émanant des établissements d'enseignement et de recherche français ou étrangers, des laboratoires publics ou privés.

\section{(c)(1)}

Distributed under a Creative Commons Attribution| 4.0 International License 


\title{
Chlamydiose et fièvre $\mathbf{Q}$ chez les ongulés de montagne : questions sur le seuil de positivité
}

\author{
E. Fromont ${ }^{1}$, D. Gauthier ${ }^{2}$, P. Gibert ${ }^{3}$, J. Hars ${ }^{4}$, M. Catusse ${ }^{5}$
}

L'épidémiologie de la faune sauvage pose des problèmes méthodologiques spécifiques : l'accessibilité des individus limite fortement les investigations possibles et conduit à une utilisation fréquente de méthodes indirectes comme la sérologie. Mais ces méthodes simples manquent de valisation externe.

En prenant l'exemple de deux maladies abortives des ongulés, nous avons recherché si les méthodes sérologiques employées seules peuvent être interprétables, et si leur utilisation permet de donner des indications sur l'évolution d'un foyer infectieux. L'objectif était de rechercher un seuil de positivité éventuel pour les deux infections et, dans chacune des espèces, d'analyser les fluctuations des deux infections dans le temps et dans l'espace.

Méthode : Les données analysées ont été récoltées au cours du suivi à long terme du statut sanitaire des chamois, isards, bouquetins et mouflons dans plusieurs massifs français, constituant une base totale de 2582 individus. Les animaux ont été testés par la méthode de fixation du complément (norme Afnor 2000).

Dans la mesure où un seul test est pratiqué, il n'est pas possible d'utiliser une validation croisée, ni sur un " gold standard " ni sur une quelconque autre méthode [1]. Nous proposons une analyse basée sur les méthodes d'analyse multivariée de tables de contingence : analyse factorielle des correspondances (AFC) et ses variantes $[2,3]$. Les différents titres sérologiques sont traités comme autant de classes d'individus, dont on analyse la distribution en fonction de variables explicatives. Afin de valider cette approche dans la détection des faux positifs, nous avons tout d'abord réalisé une étude par simulation d'une classe sérologique de faux positifs. L'analyse a ensuite été appliquée à la recherche du patron épidémiologique des deux infections.

\section{Résultats}

- Simulation de faux positifs et recherche d'un seuil de positivité : Dans la population de chamois des Bauges, nous avons créé de manière aléatoire des faux positifs. L'AFC montre que la distribution de ces faux positifs est proche de celle des négatifs, et opposée à celle de toutes les autres classes sérologiques. Ce test valide l'utilisation de l'AFC dans la recherche des faux positifs seulement si ceux-ci sont répartis de manière aléatoire. Lorsque cette démarche est appliquée aux données, on repère un seul cas probable de faux positifs concernant la chlamydiose chez le bouquetin. Dans tous les autres cas, tous les titres observés sont structurés selon deux à trois niveaux : les sérologies faibles (10 et 20$)$, moyennes $(40)$ et fortes $(\geq 80)$ apportent des informations indépendantes.
- Effet espace et effet temps : L'utilisation de l'AFC de manière " naïve " a permis de séparer les populations étudiées en trois groupes, présentant un profil « épidémique », " endémique " ou " indemne ». Ces résultats sont très concordants avec les connaissances disponibles par ailleurs sur l'exposition des populations. La même analyse permet de suivre les infections dans le temps dans certaines populations. Cette méthode a permis de retrouver des foyers qui étaient connus au plan clinique, mais aussi de détecter un important accident sérologique de chlamydiose chez le chamois des Bauges.

Discussion: La méthode proposée permet des résultats internes cohérents. Sans " gold standard », elles permettent de qualifier le statut épidémiologique d'une population. Cette approche pourrait être particulièrement utile en épidémiologie de la faune sauvage où les techniques d'analyse sont fortement contraintes.

\section{RÉFÉRENCES}

1 Enoe C, Georgiadis MP, Johnson WO. Estimation of sensitivity and specificity of diagnostic tests and disease prevalence when the true disease state is unknown. Prev Vet Med 2000 ; $45: 61-81$

2 Lebart L. Exemple d'analyse d'un tableau dont l'une des colonnes a un poids prédominant. Les Cahiers de l'Analyse des Données $1979 ; 4: 417-22$.

3 Tillard E, Faye B. Analyse d'un tableau de taux - Exploration multidimentionnelle de la circulation des agents infectieux pneumotropes chez les petits ruminants au Sénégal. In : Programmathèque ADE-4, analyses multivariées et expression graphique des données environnementales, fascicule 5 , Institut d'analyse des systèmes biologiques et socio-économiques, Université Claude-Bernard Lyon I ; 1995. p. 1-50.

http : Ilpbil.univ-lyonl.fr

'UMR CNRS 5558, université Lyon I, 43, bd du 11 novembre 1918, 69622 Villeurbanne cedex ; ${ }^{2}$ laboratoire départemental d'analyses vétérinaires de la Savoie, 321, chemin des Moulins, 73024 Chambéry cedex; ${ }^{3}$ CNERA faune de montagne ; ${ }^{4}$ unité suivi sanitaire de la faune ; ${ }^{5}$ CNERA faune de montagne, Office national de la chasse et de la faune sauvage, 8 , impasse Champ-Fila, 38320 Eybens, France. 